\title{
NOTICIAS
}

\section{Perspectivas de investigación-acción contra el consumo de tabaco en México. La reunión interinstitucional de Cocoyoc}

Durante octubre de 1999, la Dirección General de Epidemiología de la Secretaría de Salud de México, el Instituto Nacional de Salud Pública y el Institute for Global Tabacco Control, auspiciaron un encuentro de instituciones de prestación de servicios, de organismos gubernamentales y de grupos multidisciplinarios de investigación para establecer las premisas básicas de investigación-acción contra el consumo de tabaco en México, en tres vertientes principales.

Muchas de las recomendaciones pueden considerarse en contextos similares de América Latina, y ser útiles para establecer planes de acción básicos tendientes a desalentar el tabaquismo, desde la perspectiva legal, educativa y de investigación. A continuación, se reseñan los resultados de este trascendental encuentro.

\section{Grupo de Investigación}

\section{Propuesta uno de investigación}

Selección de indicadores de impacto y proceso para evaluar las activi- dades del Programa de Prevención y Control del Tabaquismo y el curso de la epidemia

1. Propuesta

Identificación de indicadores para vigilar el curso de la epidemia de tabaquismo y evaluar el impacto de las diferentes acciones de prevención realizadas dentro del Programa de Prevención y Control del Tabaquismo 1998-2000.

2. Justificación

Se considera que el Programa de Prevención y Control del Tabaquismo debe contar con indicadores que permitan documentar y evaluar el impacto de las diferentes intervenciones.

Actualmente se cuenta con información originada de fuentes diversas (encuestas, estadísticas vitales, estudios de investigación) la cual permite evaluar parcialmente las tendencias a corto, mediano y largo plazo de la epidemia. Se considera que la información existente es de calidad variable y que no se recaba de manera sistemática, ni con la periodicidad necesaria para realizar una evaluación o vigilancia adecuada. Se realizará un análisis previo de los métodos que se emplearán para obtener la información y conformar los indicadores.
3. Productos

a) Indicadores confiables por medio de los cuales se pueda evaluar el curso de la epidemia, así como el impacto de las diferentes intervenciones realizadas por el Programa, tanto en el ámbito nacional como local (estatal)

b) Un documento técnico de análisis y propuesta de indicadores

c) Plan de operacionalización

d) Plan de explotación y análisis de información.

4. Tipos de indicadores

a) Curso de epidemia (Dirección General de Epidemiología)

b) Indicadores de proceso de instrumentación de las acciones del Programa (Consejo Nacional contra las Adicciones, $\mathrm{Co}^{-}$ nadic)

c) Estrategia de la industria (respuesta de la industria tabacalera o farmacéutica; Conadic)

d) Indicadores económicos (Instituto Nacional de Salud Pública, INSP).

La caracterización de los indicadores incluirá los siguientes rubros (ejemplo)

a) Quién genera la información

b) Fuentes de información

c) Periodicidad 
d) Nivel de desagregación (estatal versus nacional)

e) Homogeneidad en el reporte

f) Costos de obtención y posibles fuentes de financiamiento.

5. Ejemplos de indicadores

I. Tabaquismo activo. Tendencias en la prevalencia de fumadores en gente joven

i. Examen de ingreso a Bachilleres

ii. Tendencias en la prevalencia de fumadoras en mujeres embarazadas

iii. Tendencias en la prevalencia de fumadores en médicos que ingresan al programa de residencia

iv. Cambios en la edad de inicio

v. Porcentaje de jóvenes que experimentan con tabaco

vi. Número de clínicas de tabaquismo, número de pacientes que ingresan, deserciones y ex fumadores en las clínicas de tabaquismo

vii. Tendencia de las enfermedades asociadas al consumo de tabaco

viii. Prevalencia en estudiantes (UNAM, IPN, otros).

\section{Tabaquismo pasivo}

i. Porcentaje de hogares en los que los padres fuman

ii. Exposición en casa y en el trabajo

iii. Encuestas sobre niveles de cotinina en niños.

III. Anuncios que promueven el tabaco.

IV. Gastos de publicidad para tabaco.

V. Cumplimiento de reglamentos

i. Encuestas sobre conocimiento

ii. Estudios sobre venta a menores de edad.
VI. Tipos y número de investigaciones por año.

6. Instituciones involucradas Mediante esta propuesta se propone convocar a un grupo de expertos, así como de representantes de las diferentes instituciones que participan en la generación o sistematización de información, para conformar un grupo de trabajo y discusión en este tema.

Instituciones responsables

- DGE (Indicadores del curso de la epidemia)

- Conadic (Indicadores de proceso)

- INSP (Indicadores económicos).

Instituciones participantes

- Dirección de Control Sanitario de la Publicidad

- Institutos Nacionales de Salud

- IMSS

- ISSSTE

- Academia de Medicina

- Asociaciones de médicos

- UNAM

- IPN

- INEGI

- SEP

- Representación estatal

- Compañías farmacéuticas.

Propuesta dos de investigación

Centro de Documentación e Información sobre Tabaco

1. Propuesta

Se propone la creación de un Centro de Documentación e Información sobre Tabaco

El objetivo principal del Centro será
i. Acopio
ii. Análisis bibliográfico
iii. Distribución

iv. Aumentar la accesibilidad a diferentes usuarios (médicos, estudiantes, etcétera).

\section{Justificación}

La información sobre tabaco se encuentra dispersa en numerosas instituciones. En general, esta información no está sistematizada, lo que dificulta su utilización oportuna. Además, no existe un registro histórico que permita hacer revisiones o búsquedas, en especial de documentos "oficiales" no publicados en revistas indexadas.

3. Productos

La creación de un Centro Nacional de Documentación e Información sobre Tabaquismo que mantenga información de fuentes internacionales y nacionales con representación estatal.

4. Instituciones involucradas Institución responsable

- Conadic

Instituciones participantes

- Todas las instituciones que realizan actividades sobre tabaquismo.

\section{Cómo}

- Identificar fondos para realizar una propuesta con términos de referencia muy específicos

- Identificar experiencias similares en la SSA (CRIDIS).

Propuesta tres de investigación

Modelos de predicción sobre daños a la salud ocasionados por el tabaquismo

\section{Propuesta}

Desarrollar modelos de predicción sobre el impacto del tabaquismo en la salud de la población, basados en información relevante para México. 


\section{Justificación}

Los modelos de predicción de impacto se han desarrollado utilizando parámetros derivados de poblaciones estadounidenses. Estos indicadores no son adecuados por múltiples razones, entre las que destacan la intensidad de tabaquismo, la composición de los cigarrillos y la presencia de otros factores de riesgo como son la obesidad, la inactividad física y algunas características de la dieta.

3. Productos

- Información sobre la relación entre tabaquismo y riesgo de cáncer de pulmón, EPOC, enfermedad cerebrovascular y enfermedad cardiovascular

- Actualización de los modelos y predicciones

- Estudios de mortalidad

- Estudios de casos y controles que permitan estimar los riesgos de las diferentes enfermedades

- Información sobre los efectos del tabaquismo pasivo en la población, especialmente en niños, jóvenes y mujeres embarazadas

- Información sobre la relación entre el tabaquismo y otras adicciones.

4. Instituciones involucradas Institución responsable

- DGE

Instituciones participantes

- INSP

\section{Grupo de Prevención}

Propuesta uno de prevención

1. Propuesta

Lograr "Escuelas Normales" y de profesores de educación física que formen profesoras (es) no fumadoras (es), con actitudes favorables hacia estilos de vida saludables, libres de tabaco y con prácticas profesionales antitabáquicas

Promover el egreso de profesoras y profesores no fumadoras (es).

2. Objetivos

- Conocer la prevalencia del consumo de tabaco en los profesores y profesoras durante su proceso de formación profesional

- Promover la creación de ambientes escolares libres de humo de tabaco en las Escuelas Normales

- Proporcionar servicios de apoyo para el abandono del tabaquismo hacia los futuros profesores

- Promover la práctica didáctica antitabáquica en el ejercicio profesional rutinario del profesor

- Promover que el profesor sea un agente de cambio en la sociedad, influya en la legislación y la normatividad relativa al tabaco, así como en los planes de estudio del sistema educativo formal, con las Asociaciones de Padres de Familia y la sociedad.

3. Estrategias

- Investigación

- Coordinación interinstitucional

- Promoción de conductas saludables

- Desarrollo académico

- Educación continua

- Fortalecimiento de la práctica didáctica

- Difusión y cumplimiento de normatividad

- Creación de servicios de atención y rehabilitación.

4. Acciones de cada estrategia

I. Investigación

i. Realizar estudios de prevalencia de tabaquismo a los alumnos de primer in- greso en las Escuelas Normales

ii. Medir la frecuencia del hábito del tabaquismo entre los profesores de las Escuelas Normales

iii. Identificar prácticas tabáquicas entre los trabajadores de las escuelas formadoras de profesores

iv. Medir el impacto de las intervenciones antitabáquicas

v. Conocer actitudes de los estudiantes y personal de las escuelas acerca del tabaquismo

vi. Dar seguimiento a más largo plazo de los profesores egresados para conocer los efectos del programa.

II. Coordinación interinstitucional

i. Presentar ante los Consejos Estatales contra las Adicciones y el Conadic las medidas de intervención antitabáquica y promover el soporte institucional para el desarrollo de programas

ii. Promover la integración de una red de escuelas de las que egresen profesores (as) no fumadores

iii. Establecer la coordinación con organismos internacionales, como la Organización Panamericana de la Salud (OPS), para la obtención de certificaciones a Escuelas Libres de Humo y formadoras de profesores no fumadores.

III. Promoción de conductas saludables

i. Desarrollo de programas educativos (pláticas, talleres, sesiones de cine, semanas culturales), di- 
rigidos a estudiantes, maestros, trabajadores administrativos y población en general

ii. Promover como actividades extracurriculares de los estudiantes de Escuelas Normales su participación comunitaria para la creación de ambientes escolares libres de humo de tabaco y la promoción de estilos de vida saludables en escuelas del nivel correspondiente

iii. Promover la formación de líderes estudiantiles con actitudes antitabáquicas favorables para ejercer función de multiplicadores

iv. Desarrollar reuniones de sensibilización con las autoridades educativas

v. Promover con las mesas directivas de las Escuelas Normales el desarrollo de semanas culturales enfocadas a prevenir el tabaquismo con actividades como módulos informativos, simposios, cursos o talleres, grupos de autoayuda, paseos, eventos conmemorativos del Día Mundial sin Tabaco, etcétera

vi. Pronunciamiento público de autoridades educativas como Escuela Normal Formadora de Profesores No Fumadores

vii. Señalización y otros esquemas de mercadeo social de Escuelas Normales libres de humo de tabaco y de profesoras (es) no fumadoras (es).

IV. Desarrollo académico

i. Efectuar revisión curricular para la identificación de contenidos en las diversas materias acerca del tabaquismo

ii. Promover ante la Comisión de Revisión Curricular la modificación de contenidos del plan de estudios para la prevención del tabaquismo en la formación de profesores.

\section{Educación continua}

i. Desarrollar simposios, seminarios, talleres y otras modalidades educativas para prevenir el tabaquismo y para desarrollar en clase los temas de tabaquismo.

VI. Difusión y cumplimiento de la normatividad

i. Reproducir y difundir la normatividad y reglamentación sobre espacios libres de humo de tabaco a personal directivo y niveles de decisión en las Escuelas Normales entre el personal docente, administrativo y alumnos

ii. Promover su cumplimiento a través de la colocación de señales, establecimiento de sanciones académicas o administrativas y de denuncias

iii. Prohibir la venta y consumo de cigarros en el interior de las instalaciones del plantel escolar y de los servicios concesionados

iv. Prohibir la participación de empresas tabacaleras o comercializadoras del tabaco y sus productos en actividades culturales y sociales, eventos académicos y deportivos organizados por las Escuelas Normales o por el nivel estudiantil.
VII. Servicios de atención y rehabilitación

i. Integrar una red de servicios de apoyo para el abandono del tabaquismo

ii. Promover la creación de una clínica o centro de atención contra el tabaquismo por Escuela Normal

ii. Promover la integración de grupos de autoayuda para dejar de fumar.

5. Resumen

Influir sobre los profesores que son responsables de la instrucción de los niños y actuar cada año con alrededor de 50 niños y sus respectivos padres de familia.

6. Instituciones involucradas

- Grupo Catalizador

- SEP en los estados

- Dirección General de Escuelas Normales

- Consejos Estatales contra las Adicciones

- Grupo de Trabajo del Tratamiento del Seminario.

\section{Propuesta dos de prevención}

\section{Propuesta}

Programa para la prevención del tabaquismo con el apoyo de la Asociación Nacional de Padres de Familia.

I. Es importante la firma del convenio entre la Secretaría de Salud y la Asociación Nacional de Padres de Familia ya sancionado y en vías de firmarse (anexarle un protocolo específico de tabaco)

II. Emitir una disposición por parte de la Asociación Nacional de Padres de Familia a todos los presidentes estatales, municipales y de escuelas sobre el programa

III. Trabajar en el comité de participación social de la escuela 
y en el comité de seguridad escolar en

i. Operación mochila modificada. Se revisaría tanto en el hogar como en la escuela por parte de los padres de familia, si algún niño trae cigarros a la escuela, precisando las acciones de esta operación, así como las medidas que se tomarían en caso de encontrarse en posesión de tabaco

ii. Colocación de carteles donde se señale la prohibición de venta de cigarrillos a menores de edad

iii. Identificación de establecimientos nocivos, es decir, tiendas donde se venden cigarrillos sueltos o cigarrillos a menores de edad sin pedir identificación en 300 metros a la redonda.

VI. Marcar una secuencia de acciones

i. Fomento de la ética comercial para que no vendan cigarrillos a menores de edad

ii. Ejercer presión social a los comerciantes para que tomen conciencia

iii. Sensibilizar a las tiendas que están alrededor de la escuela en función de la nueva reglamentación

iv. Como segundo punto, apercibir al establecimiento que no cumpla con las disposiciones

v. Denunciar ante las autoridades sanitarias a los establecimientos donde se venden cigarrillos a menores de edad.

V. Intervención de la autoridad sanitaria con apercibimiento, amonestación y multa i. Operativo vial para identificación de vendedores ambulantes de cigarrillos sueltos a menores de edad en el mismo perímetro cercano a la escuela

ii. Divulgación del reglamento con la medida específica

iii. Establecimiento de pláticas del comité de participación social con los padres de familia para que no permitan que sus hijos fumen y no fumen ellos frente a sus hijos.

Pugnar por la autorización en México del uso de la metodología de cliente simulado para hacer verificaciones sanitarias y analizar la implicación de este procedimiento en cuestión de personal, infraestructura, etcétera.

2. Metas de proceso

- Firma del convenio SSA-Asociación Nacional de Padres de Familia

- Oficio de la Asociación Nacional de Padres de Familia a las asociaciones estatales

- Elaborar la propuesta de material para difusión del "nuevo reglamento" de Control Sanitario de Productos y Servicios y de certificación de escuelas libres de humo de tabaco

- La Asociación Nacional de Padres de Familia se compromete a su reproducción

- Que los padres de familia se comprometan a sensibilizar a las tiendas para evitar la venta de cigarrillos a menores de edad

- La población objetivo son los padres de familia y a través de ellos los alumnos. Un segundo grupo son los vendedores y tiendas vecinos a la comunidad escolar
- Propuesta de padres de familia a las escuelas para la inclusión en el reglamento de escuelas libres de humo de tabaco y la certificación vía autoridades

- Establecer el tópico de tabaquismo en las Semanas Nacionales de Salud.

3. Instituciones involucradas

- Dirección General de Calidad Sanitaria de Bienes y Servicios

- Secretaría de Comercio y Fomento Industrial

- Procuraduría Federal del Consumidor

- Secretaría de Educación Pública

- Poderes locales y federales.

Propuesta tres de prevención

1. Propuesta

Unidades de salud libres de humo de tabaco

- Disposición del orden federal. Niveles ascendentes: dirección general, subsecretarios, secretario

- Disposición de orden estatal para incorporar nuevos reglamentos o disposiciones específicas

- Incorporación dentro de los sistemas de certificación incluida la iniciativa de los Hospitales Amigo del Niño y de la Madre

- Incorporarlo dentro de la Norma Oficial Mexicana de Prevención y Control del Tabaquismo en periodo de consulta pública

- Dar apoyo al personal de la unidad para que asista a tratamiento y deje de fumar

- Incorporar en la supervisión médica el cumplimiento de la normatividad

- Buscar mecanismos para incorporar la terapia de rem- 
plazo de nicotina al Cuadro Básico de Medicamentos, asî como de otras terapias farmacológicas no nicotínicas de eficacia y seguridad comprobada

- Certificación de cobertura universal del paquete básico de servicios de salud vía OPS con inclusión de atención al tabaquismo

- Promover que los alumnos, padres de familia y personal de la escuela no fume para que cubran el perfil de escuela saludable

- Formalizar la celebración del Día Mundial sin Tabaco en las escuelas (31 de mayo)

- Incorporar a la certificación de Hospital Amigo del Niño y de la Madre la condición de espacio libre de humo de tabaco

- Incluir la condición del espacio libre de humo de tabaco en los criterios de calidad de la atención

- Encuesta con la Dirección General de Estadística e Informática sobre cuántos servicios de salud tienen regulación de tabaquismo en sus instalacioneslínea basal de intervención.

\section{Grupo de legislación}

\section{Propuesta uno de legislación}

1. Propuesta

Promover la no venta a menores de edad.

2. Estrategias

- Concienciación y sensibilización con grupos objetivo como padres de familia, detallistas o vendedores, adolescentes y maestros

- Acciones de vigilancia sanitaria.

3. Responsables

- Todas las dependencias del ejecutivo, gobiernos estatales y centros educativos y sociales
- Dirección General de Calidad Sanitaria de Bienes y Servicios y autoridades locales, dentro del ámbito de su competencia

- Dirección de Control Sanitario de la Publicidad. Concienciación de detallistas o vendedores

- Coordinación con el grupo de trabajo de Prevención del Seminario.

\section{Lugar}

- Se proponen las ciudades de México y Juárez, con la finalidad de facilitar la evaluación de estos programas seis meses después.

Propuesta dos de legislación

1. Propuesta

Impulsar una política fiscal que eleve el impuesto al producto.

2. Estrategias

a) Promover seminarios o foros con autoridades hacendarias para conocer con mayor certidumbre elementos actuales de impuestos al producto

b) Contar con elementos económicos y sociales que permitan contrarrestar argumentos de autoridades hacendarias para no elevar impuestos al tabaco

c) Sensibilizar a legisladores como

i. Líderes de fracciones parlamentarias

ii. Comisión de Salud

iii. Comisión de Hacienda

d) Estimular la constitución y formación de Organizaciones No Gubernamentales para que a su vez presionen a todas las instancias involucradas para que se eleve el impuesto

e) Evaluar la repercusión económica y factibilidad de la sustitución de cultivos.

3. Responsables

- Grupo catalizador

- INSP
- Con el apoyo del Banco Mundial.

4. Lugar

a) Ejecutivo

b) Legislativo

La justificación se debe basar fundamentalmente en apoyar con recursos los siguientes programas

- Prevención

- Asistencial: padecimientos vinculados

- Subsidiar sustitución de cultivos.

Propuesta tres de legislación

1. Propuesta

Ninguna publicidad dirigida a jóvenes

Si bien se reconoce la necesidad de llegar a la prohibición total de la publicidad, actualmente es importante tener un marco regulatorio lo más restrictivo posible que limite la publicidad directa a los jóvenes.

2. Estrategias

- Vigilar que la publicidad se dé en horarios, programas y revistas para adultos

- Verificar que los contenidos no se dirijan a jóvenes, presenten situaciones aspiracionales o motivacionales

- Evaluar el impacto de las leyendas precautorias, así como la repercusión del contenido de los promocionales en los adolescentes

- Promover ante Gobernación la introducción de legislación respecto al contenido de programas en radio, televisión y cine

- Sensibilización de publicistas, medios de difusión y anunciantes para el cumplimiento de la Ley y su Reglamento

- Elaborar posibles contramensajes. 
3. Responsables

- Dirección de Control Sanitario de la Publicidad

- Conadic.

En coordinación con otros como

i. DCSP

ii. DEAJ

iii. SEGOB

iv. SEP

v. SECOFI

vi. Consejo Nacional de la Publicidad.

4. Lugar

- Medios de comunicación y grupos focales.

\section{Grupo de Tratamiento}

1. Propuesta

- Propiciar la intervención de los médicos y del personal de salud en la atención de problemas de tabaquismo, mediante la capacitación sistemática y actualizada a cargo de instituciones especializadas

- Incorporar a los integrantes de las familias, y líderes de opinión y comunitarios a las tareas de tratamiento y rehabilitación

- Incrementar el acceso y disponibilidad de medicamentos de eficacia y seguridad comprobada

- Impulsar la creación de nuevas clínicas contra el tabaquismo y desarrollar protocolos de investigación que permitan comparar los resultados de diversas modalidades terapéuticas

- Estimular la difusión masiva de los diversos tipos de tratamiento con publicidad ética

- Establecer relaciones de colaboración e intercambio con las organizaciones internacionales que desarrollan tareas de tratamiento y rehabilitación del tabaquismo.
2. Líneas de acción

Propuesta uno

- Coordinar acciones de asesoría técnica y colaboración científica con asociaciones, consejos médicos y otros grupos de profesionales de la salud

- Concertar acuerdos de colaboración y trabajo con la Academia Nacional de Medicina, la Academia Mexicana de Cirugía, la Academia de Ciencias de México, los Consejos Nacionales y Sociedades Médicas de diversas especialidades

- Llevar a cabo actividades de sensibilización con estudiantes de Facultades y Escuelas de Medicina para reducir la prevalencia de fumadores entre sus egresados

- Elaborar herramientas y materiales que apoyen la intervención del personal de salud en los distintos niveles de atención para tratar problemas relacionados con el tabaquismo

- Elaborar la Guía para la Intervención del Médico en el primer nivel de atención

- Distribuir entre el personal médico del país el Algoritmo de Intervención Médica

- Celebrar foros, encuentros y otras reuniones técnicas con especialistas y responsables del tratamiento, pertenecientes a instituciones gubernamentales y privadas

- Llevar a cabo la primera Reunión de Seguimiento a la Operación de Clínicas Contra el Tabaquismo en el año 2000

- Desarrollar acciones conjuntas con las escuelas de medicina y hospitales para transformarlos en espacios libres de humo de tabaco

- Proponer al Consejo de Salubridad General que la certificación de escuelas de medicina y hospitales considere la restricción del uso de tabaco en sus instalaciones

- Propiciar la publicación de artículos científicos, de difusión y editoriales en los órganos informativos correspondientes.

Propuesta dos

- Establecer convenios con asociaciones de padres de familia, agrupaciones sociales diversas y organizaciones no gubernamentales

- Promover la firma de convenios de colaboración (por ejemplo, con la Asociación Nacional de Padres de Familia y la Conferencia del Episcopado Mexicano, Pastoral de Salud)

- Desarrollar campañas de sensibilización, información y capacitación entre sectores sociales estratégicos que puedan apoyar las tareas de tratamiento y rehabilitación del tabaquismo

- Apoyar formas y mecanismos de divulgación entre sectores de población específicos

- Llevar a cabo pláticas de sensibilización e informativas sobre tabaquismo

- Promover la realización de foros de discusión comunitaria

- Elaborar materiales de apoyo diversos para los voluntarios que colaboren en las tareas de tratamiento y rehabilitación

- Elaboración de paquetes de información (impresos, audiovisuales)

- Realización de guías y manuales

- Capacitar al personal de las Clínicas de Tabaquismo para la realización de acciones comunitarias de promoción de la salud contra el uso del tabaco y sus consecuencias 
- Celebración de cursos y talleres

- Realización de jornadas intensivas de promoción (Día Mundial sin Tabaco, por ejemplo).

Propuesta tres

- Proponer a las instituciones del Sector Salud la adopción de medidas que amplíen las posibilidades terapéuticas en materia de tabaquismo y las pongan al alcance de la población

- Elaborar la propuesta para que se incorporen medicamentos contra el tabaquismo en los Cuadros Básicos de las instituciones del Sector Salud

- Proponer al Consejo de Salubridad General que sugiera a las farmacias de todo el país que tengan disponibles fármacos para el tratamiento del tabaquismo

- Difundir en todo el país el Algoritmo para Dependientes de Farmacias.

Propuesta cuatro

- Promover ante las autoridades de salud y grupos privados el establecimiento de nuevas Clínicas de Tabaquismo

- Enviar propuestas de apoyo técnico a cada uno de los Servicios Estatales de Salud, delegaciones del IMSS e ISSSTE de cada entidad federativa, así como a particulares y empresas reconocidas

- Llevar a cabo con periodicidad anual el Curso Nacional de Actualización para Responsables de Clínicas de Tabaquismo

- Estimular y apoyar la investigación sobre modalidades terapéuticas aplicables al tabaquismo

- Llevar a cabo un encuentro de investigadores sobre el tratamiento del tabaquismo y en- fermedades asociadas con el uso de tabaco

- Elaborar un proyecto de investigación comparativo de los resultados correspondientes a las distintas modalidades terapéuticas.

\section{Propuesta cinco}

- Proponer formas y mecanismos de participación de especialistas y otros profesionales para diseñar y llevar a la práctica campañas de propaganda y mensajes que difundan las diversas modalidades de tratamiento

- Desarrollar campañas de medios con cada una de las empresas farmacéuticas que producen o distribuyen en México medicamentos para el tratamiento del tabaquismo

- Convocar a un concurso nacional de proyectos de campañas de difusión del tratamiento y rehabilitación del fumador

- Diseñar proyectos de difusión de las modalidades terapéuticas del tabaquismo, que aprovechen espacios oficiales de radio y televisión.

Propuesta seis

- Estimular relaciones de colaboración e intercambio con organizaciones de otros países

- Establecer relaciones formales de colaboración con diversas sociedades, por ejemplo, con la Society for Research on Tobacco and Nicotine y la American Society of Addiction Medicine.

Colaboración con el Grupo de Trabajo de Prevención

3. Instituciones involucradas

- Compañías farmacéuticas

4. Fuentes de financiamiento.

- Sector privado.

\section{Grupo de Capacitación}

Propuesta uno de capacitación

1. Propuesta

Diagnóstico nacional del contenido curricular sobre temas relativos a tabaquismo de las escuelas de formación de los profesionales de salud y educación.

2. Objetivo

Identificar si en los contenidos curriculares consideran los temas de tabaquismo y en qué profundidad.

3. Meta

Contar con la información en seis meses.

4. Institución responsable Clínicas de Tabaquismo del INER.

5. Posibles fuentes de financiamiento Johns Hopkins, OPS, UNICEF.

\section{Propuesta dos de capacitación}

\section{Propuesta}

Inclusión en el Programa Nacional de Actualización para el Médico General (Pronadameg) un capítulo sobre prevención, tratamiento y rehabilitación en tabaquismo.

2. Objetivo

Que el médico general tenga capacitación y actualización sistematizada y homogénea en prevención, tratamiento y rehabilitación en tabaquismo.

3. Meta

Que cada año se actualice a $\mathrm{n} \%$ de los médicos generales.

4. Población blanco

Médicos generales.

5. Contenidos

Guía de manejo del problema de casos de tabaquismo para médicos en el primer nivel de atención

6. Institución responsable Pronadameg 
7. Posibles fuentes de financiamiento

Pronadameg.

Propuesta tres de capacitación

1. Propuesta

Con periodicidad anual impartir el curso a profesionales de la salud para la atención del tabaquismo (Clínicas de Tabaquismo).

2. Objetivo

Aumentar la capacidad de respuesta a la necesidad de contar con un mayor número de Clínicas de Tabaquismo.

3. Meta

Capacitar anualmente a 100 profesionales de la salud en el manejo de clínicas de tabaquismo.

4. Población blanco

Profesionales de la salud.

5. Institución responsable

- INER

- IMSS

- CONADIC

- ISSSTE

- DIF

- Servicios médicos de la UNAM.

6. Posibles fuentes de financiamiento

- OPS

- Industria farmacéutica.

Propuesta cuatro de capacitación

1. Propuesta

Proyecto piloto de capacitación en prevención, riesgos y daños por tabaquismo, en el cuarto grado de la licenciatura de Educación Primaria y el último grado de las licenciaturas de Psicología Educativa y de Pedagogía en la Universidad Pedagógica Nacional.

2. Objetivo

Complementar la formación del educando con información preventiva sobre tabaquismo.
3. Meta

Participación de al menos un grupo de la Benemérita Escuela Nacional de Maestros y dos de la Universidad Pedagógica Nacional en el proyecto piloto.

4. Institución responsable

- Benemérita Escuela Nacional de Maestros

- Universidad Pedagógica Nacional.

5. Posibles fuentes de financiamiento

Interinstitucional de entidades afines a los Sectores Salud y Educación.

Propuesta cinco de capacitación

1. Propuesta

Difusión del manejo del "Algoritmo de la intervención médica para ayudar a la cesación tabáquica".

2. Objetivo

Difundir el material entre el personal de salud.

3. Meta

Difundir en el 2000 un mínimo de 50000 cartillas.

4. Institución responsable Grupo Catalizador.

5. Posibles fuentes de financiamiento

Industria farmacéutica.

Propuesta seis de capacitación

1. Propuesta

Incrementar la difusión del programa "Construye tu vida sin adicciones" entre el personal educativo y de salud.

2. Objetivo

Difundir el material entre el personal de salud.

3. Meta

Realizar tres concursos en el 2000.

4. Institución responsable

- INER
- IMSS

- Conadic

- ISSSTE

- DIF

- Servicios médicos de la UNAM

- SEP.

5. Posibles fuentes de financiamiento

Interinstitucional.

Propuesta siete de capacitación

1. Propuesta

Desarrollo de una página Web sobre capacitación en tabaquismo con dos grandes vertientes: personal educativo y personal de salud.

2. Objetivo

Accesibilidad a un modelo de educación electrónica

3. Meta

Un año para su desarrollo.

4. Población blanco

Personal de salud y de educación.

5. Institución responsable

- Instituto Nacional de Salud Pública

- Dirección General de Epidemiología

- Conadic.

6. Posibles fuentes de financiamiento

- Johns Hopkins

- Industria farmacéutica.

\section{La Declaración de} Cuernavaca para un ambiente libre de humo de tabaco

La Asociación Morelense de Lucha contra el Cáncer, y el Departamento de Investigación en Tabaquismo del Centro de Investigación en Salud Pública del Instituto Nacional de Sa- 
lud Pública, formularon una declaración que explica en gran medida los daños a la salud atribuibles al consumo de tabaco, así como la solicitud dirigida a las máximas autoridades de salud, de acciones institucionales. El IX Congreso Nacional de Investigación en Salud Pública, celebrado en marzo de 2001, sirvió de marco para la adherencia a tal pronunciamiento de cerca de 2000 investigadores de diversas regiones de la República Mexicana, y la solidaridad de distinguidos investigadores internacionales. La Declaración de Cuernavaca para un ambiente libre de humo de tabaco fue la siguiente:

Considerando que:

- El consumo de cigarro de tabaco es una de las causas más importantes de morbilidad y mortalidad

- Se estima que para el año 2020 habrán ocurrido 8.4 millones de muertes atribuibles al tabaco, y $70 \%$ de las víctimas serán de países en desarrollo

- Cerca de la mitad de los 500 millones de fumadores en el mundo son niños o adolescentes

- Dada la reducción en los niveles de consumo de tabaco en los países desarrollados, las compañías tabacaleras se han dirigido de manera agresiva a otros países para extender el mercado y mantener sus ganancias

- En México, el número de fumadores aumentó de 9.2 millones en 1988 a 14.3 millones en 1998, por lo que la morbilidad y la mortalidad por enfermedades atribuibles al tabaco aumentarán proporcionalmente

- El tabaco se vende debido a la dependencia química que genera en sus consumidores

- La industria tabacalera utiliza de manera intensiva la publicidad para que cada vez más jóvenes y mujeres se conviertan en fumadores, lo cual ha provocado que de los casi 1.2 miles de millones de fumadores que existen actualmente en el mundo, $200 \mathrm{mi}-$ llones sean mujeres, y que de las 4000000 de muertes que ocurren al año, cerca de 500000 sean de mujeres

- Las compañías tabacaleras han hecho esfuerzos para lograr que niños, jóvenes y mujeres fumen tabaco, como lo demuestran documentos internos de las propias compañías

- Los jóvenes son de importancia capital para esta industria, ya que es este grupo el de mayor vulnerabilidad para generar adicción, y la dinámica de mercado demanda concentrar esfuerzos para reclutar gente joven

- La publicidad propicia la motivación para fumar tabaco, asociando engañosamente valores "positivos" como independencia, buena salud, riqueza, y juventud a la acción de fumar, mientras que en realidad esta adicción provoca graves daños a la salud y acorta significativamente la esperanza de vida

- A pesar de los esfuerzos oficiales para evitar que se fume en los edificios públicos, el humo de tabaco en el ambiente continúa siendo un factor de riesgo importante para generar cáncer del pulmón, enfermedad pulmonar obstructiva crónica, enfermedad isquémica cardiovascular y otras enfermedades graves en fumadores pasivos o involuntarios

- Los desechos relacionados con los 700 millones de cajetillas de cigarros que se consumen cada año en México también son un factor de contaminación ambiental

- La adicción a la nicotina afecta directa o indirectamente la vida de todos los seres humanos, y significa considerables costos económicos y sociales

- La adicción a la nicotina destruye las convivencias sanas

- El consumo de tabaco y la adicción a la nicotina son dos facetas de un mal innecesario y evitable, pero para combatirlo es indispensable que la sociedad y el Estado Mexicano se organicen para invertir recursos humanos, financieros e institucionales en el desarrollo y la aplicación de programas accesibles a toda la población.

\section{Recomendamos}

1. Organizar un sistema de información en el ámbito estatal y municipal, para acompañar la producción, comercio y consumo del tabaco, medir con precisión la morbilidad y la mortalidad atribuibles, así como los costos de atención, y analizar las tendencias y causas de la adicción a la nicotina

2. Reunir la información y argumentos indispensables para a) evitar la iniciación en el consumo de tabaco de niños, adolescentes $\mathrm{y}$ adultos jóvenes de uno $\mathrm{u}$ otro sexo; b) contener la producción, venta y consumo de tabaco y consecuentemente disminuir o eliminar los daños a la salud ocasionados por éste; c) conseguir, por medio de programas eficaces de prevención, diagnóstico, tratamiento y vigilancia, que los adictos a la nicotina dejen de fumar tabaco

3. Instrumentar una red de recursos multi-institucionales, permanente, cuyas responsabilidades estén enfocadas en proyectos específicos de investigación-acción y desarrollo de recursos humanos en las siguientes líneas: a) prevención 
del consumo, b) tratamiento de la adicción y del síndrome de abstinencia, c) cesación y abstinencia, y e) soporte psicosocial y seguimiento para la prevención de las recaídas

Los abajo firmantes estamos de acuerdo en participar como miembros activos de la Red de Instituciones de Educación, Seguridad y Salud para el Manejo Integrado del Consumo de Tabaco y la Adicción a la Nicotina y

\section{Sugerimos}

Al señor doctor Julio Frenk Mora, Secretario de Salud, que:

I. Reconozca oficialmente la gravedad del problema de salud colectiva que representa para los mexicanos la adicción a la nicotina

II. Declare de alta prioridad el Programa Antitabáquico dentro del Programa Nacional de Salud, y asigne el presupuesto requerido para iniciar las actividades que permitan alcanzar las siguientes metas a corto plazo:

- Incrementar el impuesto al tabaco y conseguir que una parte proporcional de los ingresos que se recaudan en este concepto se asignen a la partida presupuestaria correspondiente al Programa Antitabáquico

- Eliminar la publicidad de los productos de tabaco en todos los medios de difusión masiva

- Incluir leyendas de alarma en las cajetillas de cigarros, cigarrillos y empaques de otros productos de tabaco sobre el peligro para la salud y la vida de las personas que los consumen

- Abogar por la promulgación de modificaciones a la Ley de Salud, reglamentos y normas específicas, para evitar la contaminación con humo de tabaco en el ambiente y para la protección de los fumadores pasivos y fumadores involuntarios
- Promover vigorosamente el desarrollo de clínicas para la cesación del hábito de fumar en todos los adictos a la nicotina

- Organizar e instrumentar programas de educación para la salud que consigan hacer comprender a la población, especialmente a niños, jóvenes y mujeres, el riesgo de fumar tabaco y la manera de eliminarlo.

III.Coordine las actividades de la Red para el Manejo Integrado del Consumo de Tabaco y de la Adicción a la Nicotina, en colaboración con las instituciones del Estado Mexicano

IV.Promueva la decisión política del Ejecutivo Federal para la participación de México en el Control, Prevención y Vigilancia Mundial del Tabaco en el Mundo, en los términos del Convenio Marco de la Organización Mundial de la Salud. 